ORIGINAL

\title{
Efecto de Debaryomyces hansenii en la respuesta antioxidante de juveniles de camarón blanco Litopenaeus vannamei
}

\section{Effect of Debaryomyces hansenii on the antioxidant response of juvenile white shrimp Litopenaeus vannamei}

\author{
María Pacheco M, ${ }^{1}$ M.Sc, Ángel Campa C, ${ }^{1}$ Ph.D, Gabriel Aguirre G, $2 *$ Ph.D, \\ Antonio Luna G, ${ }^{3}$ Ph.D, María Guzmán M, ${ }^{1}$ Ph.D, Felipe Ascencio, ${ }^{1}$ Ph.D.
}

\begin{abstract}
${ }^{1}$ Centro de Investigaciones Biológicas del Noroeste, Mar Bermejo No. 195, Col. Playa Palo de Santa Rita, La Paz, BCS, México. 2Universidad Autónoma de Tamaulipas. Facultad Facultad de Medicina Veterinaria y Zootecnia. Km. 5 Carr. Cd. Victoria - Mante, Cd. Victoria, Tamps, México. ${ }^{3}$ Centro Interdisciplinario de Investigación para el Desarrollo Integral Regional-Instituto Politécnico Nacional. Blvd Juan de Dios Bátiz Paredes 250, Guasave, Sin., México. *Correspondencia: gabaguirre@uat. edu. $\mathrm{mx}$.
\end{abstract}

Recibido: Enero de 2011; Aceptado: Agosto de 2011.

\section{RESUMEN}

Objetivo. Determinar la respuesta antioxidante [actividad de superóxido dismutasa (SOD) y catalasa (CAT) ] así como la cuenta total de hemocitos (CTH) y el contenido de proteínas (CP) en camarones (Litopenaeus vannamei) expuestos a diferentes dosis y cepas de la levadura Debaryomyces hansenii (DH5, DH6, LL1), y un inmunoestimulante comercial (LAM). Materiales y métodos. Las levaduras fueron cultivadas y suministradas diariamente en concentraciones diferentes (104 - 106 UFC/ $\mathrm{mL}$ ) directamente a los tanques de cultivo de los camarones $(8 \pm 0.2 \mathrm{~g})$ mientras que LAM fue aplicado una vez a la semana $(0.5 \mathrm{mg} / \mathrm{L})$. Los organismos fueron mantenidos bajo condiciones de laboratorio $\left(28^{\circ} \mathrm{C}, 35 \%, 80 \%\right.$ de recambio diario de agua, dieta comercial para camarón ad libitum). Los tratamientos fueron distribuidos por duplicado y los resultados evaluados a los 15 días con un análisis de varianza y una prueba de Tukey. Resultados. Se registró un CTH significativo $(p<0.05)$ en los tratamientos con DH6 y LL1 (106 UFC/mL) comparada con el control, mientras que las cepas DH5 y DH6 revelaron un incremento significativo $(p<0.05)$ de CP con la dosis de $104 \mathrm{UFC} / \mathrm{mL}$. Los camarones tratados con LAM incrementaron significativamente $(p<0.05)$ los valores de SOD y CAT. Conclusiones. Los resultados obtenidos demuestran que $D$. hansenii incrementa la respuesta antioxidante y $\mathrm{CTH}$ en camarones.

Palabras clave: Debaryomyces hansenii, inmunoestimulación, Litopenaeus vannamei (Source: CAB). 


\begin{abstract}
Objective. To determine the antioxidant response [superoxide dismutase (SOD) and catalase (CAT) activity], as well as the total haemocyte count (CTH), and protein level (CP) in shrimp Litopenaeus vannamei exposed to different doses and yeast strains of Debaryomyces hansenii (DH5, DH6, LL1), and a commercial immunostimulant (LAM). Materials and methods. The yeast strains were cultured and administered daily directly into the shrimp tanks $(8 \pm 0.2 \mathrm{~g})$ in different concentrations $(104-106 \mathrm{CFU} / \mathrm{mL})$, while the LAM was administered weekly $(0.5 \mathrm{mg} / \mathrm{L})$. The organisms were kept under laboratory conditions $\left(28^{\circ} \mathrm{C}, 35 \%, 80 \%\right.$ daily water exchange, commercial diet for shrimp fed ad libitum). Each treatment was twice distributed and the results were evaluated through variance analysis and the Tukey method. Results. A significant THC $(p<0.05)$ was detected for the treatments with DH6 and LL1 (106 CFU / $\mathrm{mL}$ ) compared to the control group, whereas strains DH5 and DH6 presented a significant increase $(p<0.05)$ in the value of CP for the $104 \mathrm{CFU} / \mathrm{mL}$ dose. SOD and CAT values increased significantly $(p<0.05)$ for shrimp exposed to LAM. Conclusions. The results of this study illustrate that $D$. hansenii increased the antioxidant response, and CTH of shrimp.
\end{abstract}

Key words: Debaryomyces hansenii, immunostimulation, Litopenaeus vannamei (Source: CAB).

\title{
INTRODUCCIÓN
}

Como todo invertebrado, el sistema inmune de los camarones peneidos está mediado por hemocitos (hialinos, granulares y semigranulares) los cuales poseen capacidad citotóxica y de comunicación intercelular, lo que les facilita realizar funciones de reconocimiento de antígenos, coagulación, fagocitosis, melanización, formación de nódulos y encapsulación (1). El sistema inmune de los camarones cuenta también con la presencia de varios efectores plasmáticos (péptidos antimicrobianos, histonas, enzimas lisosomales, proteínas de reconocimiento de glucanos y lipopolisacáridos), sistema profenoloxidasa, y la cascada de coagulación que favorece la destrucción de los patógenos $(1,2)$.

Para activar el sistema inmune de los camarones y describir los mecanismos de funcionamiento del mismo se han empleado microorganismos y/o sus derivados (B-glucano, laminarina, lipopolisacáridos, y péptidoglucanos) (3). El suministro de estos productos incluido en el alimento o aplicados en forma directa (inyección, inmersión, bio-encapsulación, o intubación) sugiere que pueden ser usados como una medida de protección en contra de los patógenos que afectan al camarón (4).

Levaduras como Candida sake, C. tropicalis, Debaryomyces hansenii, Rhodotorula rubra, $R$. glutinis, Saccharomyces cerevisiae, y Schizophyllum commune han sido empleadas como activadores del sistema inmune del camarón (Fenneropenaeus indicus, Penaeus monodon) y peces (Channa striata, Sparus aurata) en contra de agentes patógenos.
Miles et al (5) observó que los bio-productos derivados de levadura mejoran la respuesta inmunológica de Ch. striata en contra de Aphanomyces invadans ( $A$. piscicida) causante del síndrome epizoótico ulcerativo en estos peces. Los organismos alimentados o expuestos a las levaduras y/o sus derivados fueron menos susceptibles a bacterias oportunistas y virus y además presentaron una mejor supervivencia y crecimiento. Esto resultados sugieren la posibilidad de usar estos microorganismos como inmuno-estimulantes y fuente de bio-productos empleados para ese fin (6-9).

En los crustáceos, muchos mecanismos de defensa celulares dependen de la producción controlada de radicales libres (10), como respuesta al estrés oxidativo, en donde el metabolismo aerobio de los crustáceos genera sustancias reactivas al oxígeno que son eliminadas por un sistema de defensa antioxidante que incluye a las enzimas superóxido dismutasa (SOD), catalasa (CAT) y peroxidasa $(10,11)$. Estos metabolitos también están involucrados en el control de agentes patógenos, como en la fagocitosis de Vibrio harveyi que desencadena un incremento de la SOD (10, 12). Reyes-Becerril et al (7) proporcionaron a cabrilla sardinera (Mycteroperca rosacea) una dieta enriquecida con $D$. hansenii (106 UFC/g) para estimular su respuesta inmune durante el cultivo de este organismo en un medio con dinoflagelados patógenos (Amyloodinium ocellatum). Los organismos alimentados con esta dieta presentaron una sobrevivencia significativa mayor comparada con el grupo control, mostrando además niveles 
significativos en la actividad de la enzima SOD y una relación importante de este parámetro con la actividad de la enzima CAT. En S. aurata también aumentó la actividad de la SOD al ser alimentados con una dieta enriquecida con $D$. hansenii (106 UFC/g)(8).

El objetivo del presente trabajo fue determinar la respuesta antioxidante [actividad de superóxido dismutasa (SOD) y catalasa (CAT)] así como la cuenta total de hemocitos (CTH) y el contenido de proteínas (CP) en camarones (Litopenaeus vannamei) expuestos a diferentes dosis y cepas de la levadura Debaryomyces hansenii (DH5, DH6, LL1), y un inmunoestimulante comercial (LAM).

\section{MATERIALES Y MÉTODOS}

\section{Cepas de microorganismos e inmunoesti-} mulantes. Las tres cepas experimentales fueron obtenidas de la colección de levaduras del Centro Investigaciones Biológicas del Noroeste (CIBNOR). Dos de las cepas utilizadas fueron aisladas del medio marino [Debaryomyces hansenii (DH5 y DH6)] y la otra de cítricos $[D$. hansenii (LL1)]. Previo a los bioensayos, las cepas mantenidas por crio-conservación $\left(-80^{\circ} \mathrm{C}\right)$ fueron descongeladas y cultivadas en placas con agar YPD ( $2 \%$ dextrosa, $2 \%$ peptona, $1 \%$ extracto de levadura, $2 \%$ agar) a $30^{\circ} \mathrm{C}$ por $24 \mathrm{~h}$. Las colonias fueron extraídas del agar y suspendidas en tubo de ensayo con $10 \mathrm{~mL}$ de agua de mar estéril (filtrada con filtros con retención de partículas poro de $0.2 \mu \mathrm{m}$ ), la suspensión de levaduras fue concentrada hasta alcanzar una absorbancia de 1.0 a una densidad óptica de $540 \mathrm{~nm}$ (colorímetro, Linson 3) equivalente a $1 \times 10^{9} \mathrm{UFC} / \mathrm{mL}$. Además, se empleó laminarina (LAM) extraída de la macroalga Laminaria digitata (Sigma, L-9634) como inmunoestimulante comercial.

Bioensayos. Se usaron dos lotes de camarones juveniles de $L$. vannamei $(8 \pm 0.2 \mathrm{~g})$ provenientes del laboratorio de larvicultura del CIBNOR. Los organismos fueron aclimatados durante 15 días en tanques de fibra de vidrio de $1500 \mathrm{~L}$ con agua de mar filtrada $(5 \mu \mathrm{m})$ a $28^{\circ} \mathrm{C}$ y aireación continua. Los camarones fueron alimentados ad libitum mañana y tarde (9:00 y 17:00 h) diariamente con una dieta comercial (PIASA, $35 \%$ de proteína).

El primer bioensayo tuvo una duración de 15 días. Los organismos experimentales fueron seleccionados al azar y distribuidos en tinas de fibra de vidrio de $60 \mathrm{~L}$ (7 camarones/tina) con agua de mar filtrada $(5 \mu \mathrm{m})$ a $28^{\circ} \mathrm{C}$ y aireación constante. Los tratamientos se hicieron por duplicado. La alimentación diaria fue ad libitum con la dieta comercial y horario antes señalados (13). Mediante sifoneo se eliminó diariamente la materia particulada del fondo de las tinas, recuperando enseguida el volumen de agua perdido ( $80 \%)$. La temperatura del agua se registró diariamente a la misma hora. Además, los organismos estuvieron sujetos a fotoperiodo natural. Los inmunoestimulantes se inocularon en el agua del sistema de cultivo de acuerdo con los siguientes tratamientos: 1 ) Control, sin tratamientos; 2 y 3$) \mathrm{DH} 5\left(1 \times 10^{4}\right.$, y $1 \times 10^{6} \mathrm{UFC} / \mathrm{mL}$, respectivamente); 4 y 5) DH6 (1) $10^{4}$, y $1 \times 10^{6} \mathrm{UFC} / \mathrm{mL}$, respectivamente); 6) LAM $(0.20 \mathrm{mg} / \mathrm{mL})$. Las dosis de levaduras suministradas estuvieron de acuerdo a las reportadas por Reyes-Becerril et al (7). Las dosis de laminarina se basaron en el trabajo de Campa-Córdova et al (11). La inoculación de los tratamientos en el agua de cultivo se repitió después de cada recambio. El segundo experimento tuvo la misma duración y condiciones de cultivo que se señalaron en el primer bioensayo. Los tratamientos utilizados fueron los siguientes: 1) Control, sin inmunoestimulante; 2,3 , y 4) LL1 (1x104, $1 \times 10^{5}$ y $1 \times 10^{6} \mathrm{UFC} / \mathrm{mL}$ respectivamente); 5) LAM $(0.20 \mathrm{mg} / \mathrm{mL})$ adicionada cada siete días.

Extracción de la hemolinfa. La hemolinfa se obtuvo en el periodo de intermuda y se extrajo con jeringas para insulina ( $27 \mathrm{G} \times 13$ $\mathrm{mm}$ ) a partir de la región ventral del primer segmento abdominal. La jeringa se cargó con una solución isotónica para camarón y EDTA como anticoagulante (SIC- EDTA- $\left.\mathrm{Na}_{2}\right)(\mathrm{NaCl}$ $450 \mathrm{mM}, \mathrm{KCl} 10 \mathrm{mM}$, Hepes $10 \mathrm{mM}$, EDTA-Na $10 \mathrm{mM}, \mathrm{pH} 7.3,850 \mathrm{mOsm} / \mathrm{kg}$ ) previamente enfriada a $4^{\circ} \mathrm{C}(15,16)$ en una proporción $2: 1$ ( 2 volúmenes de SIC-EDTA por cada volumen extraído de hemolinfa) (11).

Conteo total de hemocitos (CTH). EI CTH se realizó conforme a la técnica descrita por Campa-Córdova et al (11) en donde se aplican $400 \mu \mathrm{L}(1: 4)$ de formaldehido al $4 \%$ a $100 \mu \mathrm{L}$ de hemolinfa con anticoagulante. Los hemocitos se cuantificaron en una cámara Neubauer, utilizando un microscopio óptico (400x).

Cuantificación de proteína (CP). La CP en los hemocitos provenientes de la hemolinfa $(\mathrm{mg} / \mathrm{mL})$ se determinó en un lector de placa y una prueba de micro-determinación de proteína (Bio-Rad, USDA) realizándose la lectura a una longitud de onda de $595 \mathrm{~nm}$ (8). Se efectuó una curva a partir de los reactivos estándares que posee la prueba antes señalada. 
Actividad de la superóxido dismutasa (SOD) y catalasa (CAT). La SOD se determinó por el método descrito por ReyesBecerril et al $(7,8)$; Campa-Córdova et al (11). Se utilizó NBT en presencia de riboflavina. Se colocaron $10 \mu \mathrm{L} v / \mathrm{v}$ de hemolinfa y buffer fosfato de potasio (50 mM, pH 7.8) con $200 \mu \mathrm{L}$ de la mezcla de reacción ( $0.1 \mathrm{mM}$ EDTA, $13 \mu \mathrm{M}$ metionina, $0.75 \mathrm{mM}$ NBT, $20 \mu \mathrm{M}$ riboflavina, $50 \mathrm{mM}$ buffer de fosfato, pH 7.8). Esta solución fue expuesta a luz fluorescente (1 min) o cuando el control lograra una densidad óptica de 0.2-0.25 a $570 \mathrm{~nm}$.

La actividad CAT se evaluó con una prueba comercial (Sigma, C-9284). El método determina el decremento de la concentración de peróxido de hidrógeno a una longitud de onda de $240 \mathrm{~nm}$ y un coeficiente de extinción de $40 / \mathrm{M} / \mathrm{cm}$. Se consideró que una unidad de CAT descompone $1 \mu \mathrm{mol}$ de $\mathrm{H}_{2} \mathrm{O}_{2} / \mathrm{min}$ en $50 \mathrm{mM}$ de buffer fosfato a pH 7 ajustando la absorbancia de la solución a $0.5 \pm 0.01$. La actividad de CAT fue expresada en términos de unidades ( $\mu$ moles de substrato transformado a producto/min) por miligramo de proteína.

Análisis estadístico. Los resultados obtenidos fueron analizados por medio de un análisis de varianza (ANOVA) de una vía para detectar diferencias entre tratamientos $(p<0.05)$. Se realizó la prueba de Tukey (HSD) para identificar diferencias $(p<0.05)$, usando el software Statistica (Versión 6.0).

Tabla 1. Número de hemocitos, contenido de proteína, actividad superóxido dismutasa (SOD) y catalasa en juveniles de camarón blanco $L$. vannamei expuestos a diferentes cepas y dosis de $D$. hansenii o inmunoestimulante comercial.

\begin{tabular}{|c|c|c|c|c|}
\hline & \multicolumn{4}{|c|}{ Bioensayo 1} \\
\hline & $\begin{array}{c}\text { Conteo de } \\
\text { hemocitos } \\
(1 \times 106 \\
\text { cel/ml })\end{array}$ & $\begin{array}{c}\text { Contenido } \\
\text { de proteína } \\
(\mathrm{mg} / \mathrm{ml})\end{array}$ & $\begin{array}{l}\text { Actividad } \\
\text { de SOD } \\
(U / \mathrm{mg})\end{array}$ & $\begin{array}{c}\text { Actividad } \\
\text { catalasa } \\
\text { (U/mg) }\end{array}$ \\
\hline Control & $\begin{array}{c}5.10 \\
\pm 0.30\end{array}$ & $\begin{array}{c}1.70 \\
\pm 0.09\end{array}$ & $\begin{array}{l}35.29 \\
\pm 1.75\end{array}$ & $\begin{array}{l}3087.30 \\
\pm 197.33\end{array}$ \\
\hline DHA & $\begin{array}{c}7.59 \\
\pm 1.37\end{array}$ & $\begin{array}{c}3.30 \\
\pm 0.21 *\end{array}$ & $\begin{array}{c}23.89 \\
\pm 2.06^{*}\end{array}$ & $\begin{array}{l}1920.38 \\
\pm 89.28 *\end{array}$ \\
\hline DHB & $\begin{array}{c}7.54 \\
\pm 1.21\end{array}$ & $\begin{array}{c}2.21 \\
\pm 0.37\end{array}$ & $\begin{array}{l}31.00 \\
\pm 3.91\end{array}$ & $\begin{array}{l}2674.02 \\
\pm 372.27\end{array}$ \\
\hline $\mathrm{DHC}$ & $\begin{array}{c}5.95 \\
\pm 0.16\end{array}$ & $\begin{array}{c}2.34 \\
\pm 0.14^{*}\end{array}$ & $\begin{array}{l}30.34 \\
\pm 4.06\end{array}$ & $\begin{array}{l}2697.79 \\
\pm 386.80\end{array}$ \\
\hline DHD & $\begin{array}{c}13.14 \\
\pm 1.75^{*}\end{array}$ & $\begin{array}{c}1.86 \\
\pm 0.14\end{array}$ & $\begin{array}{l}29.33 \\
\pm 8.12\end{array}$ & $\begin{array}{l}2677.34 \\
\pm 258.01\end{array}$ \\
\hline Laminarina & $\begin{array}{c}7.40 \\
\pm 1.05\end{array}$ & $\begin{array}{c}1.33 \\
\pm 0.26\end{array}$ & $\begin{array}{c}45.86 \\
\pm 3.43^{*}\end{array}$ & $\begin{array}{c}3980.32 \\
\pm 346.32 *\end{array}$ \\
\hline
\end{tabular}

Bioensayo $1=$ DH5 y DH6 $\left(10^{4}\right.$ o $\left.10^{6} \mathrm{UFC} / \mathrm{mL}\right)$ y Laminarina $(0.05 \mathrm{mg} / \mathrm{L})$. $(*)$ denota diferencias significativas respecto al control $(p<0.05)$.

\section{RESULTADOS}

Conteo total de hemocitos (CTH). El CTH obtenido en el presente trabajo tras la aplicación de las diferentes cepas y dosis de levaduras se observa en las tablas 1 y 2 . Se detectó un incremento significativo $(p<0.05)$ en los tratamientos con la dosis de $1 \times 10^{6}$ UFC/mL de las cepas DH6 $\left(14 \times 10^{6}\right.$ hemocitos $/ \mathrm{mL})$ y LL1 $\left(6 \times 10^{6}\right.$ hemocitos $\left./ \mathrm{mL}\right)$ en comparación con los grupos controles $\left(3-4 \times 10^{6}\right.$ hemocitos $/ \mathrm{mL}$ ). La laminarina y demás dosis de levadura no generaron incrementos significativos de $\mathrm{CTH}$ comparados con sus respectivos grupos control (Tabla 1 y 2 ).

Cuantificación de proteína (CP). El contenido de proteína de los hemocitos provenientes de juveniles de $L$. vannamei expuestos a la dosis de $1 \times 10^{4} \mathrm{UFC} / \mathrm{mL}$ de $D$. hansenii de origen marino (DH5 y DH6) reveló valores significativamente mayores $(p<0.05)$ que el grupo control (Tabla 1$)$. Los camarones expuestos a la cepa LL1 revelaron fluctuaciones de proteína en los hemocitos en sus diferentes dosis de aplicación, sin diferencias significativas con su respectivo grupo control (Tabla 2).

Actividad de la superóxido dismutasa (SOD) y catalasa (CAT). La actividad SOD en hemocitos de L. vannamei expuestos a la laminarina en el bioensayo 1 revela valores significativamente superiores comparados con el grupo control (Tabla 1). Contrariamente, los organismos expuestos la dosis de $1 \times 10^{4} \mathrm{UFC} / \mathrm{mL}$ de DH5 mostraron valores significativos inferiores

Tabla 2. Número de hemocitos, contenido de proteína, actividad superóxido dismutasa (SOD) y catalasa en juveniles de camarón blanco $L$. vannamei expuestos a diferentes cepas y dosis de $D$. hansenii o inmunoestimulante comercial.

\begin{tabular}{|c|c|c|c|c|}
\hline & \multicolumn{4}{|c|}{ Bioensayo 2} \\
\hline & $\begin{array}{c}\text { Conteo de } \\
\text { hemocitos } \\
(1 \times 106 \\
\text { cel/ml })\end{array}$ & $\begin{array}{c}\text { Contenido } \\
\text { de proteína } \\
(\mathrm{mg} / \mathrm{ml})\end{array}$ & $\begin{array}{l}\text { Actividad } \\
\text { de SOD } \\
\text { (U/mg) }\end{array}$ & $\begin{array}{c}\text { Actividad } \\
\text { catalasa } \\
(\mathrm{U} / \mathrm{mg})\end{array}$ \\
\hline Control & $\begin{array}{c}3.00 \\
\pm 0.52\end{array}$ & $\begin{array}{c}3.03 \\
\pm 0.51\end{array}$ & $\begin{array}{l}11.90 \\
\pm 7.59\end{array}$ & $\begin{array}{l}1635.77 \\
\pm 291.91\end{array}$ \\
\hline $\operatorname{LL} 1\left(10^{4}\right)$ & $\begin{array}{c}2.36 \\
\pm 0.41\end{array}$ & $\begin{array}{c}2.24 \\
\pm 0.83\end{array}$ & $\begin{array}{l}17.99 \\
\pm 2.76\end{array}$ & $\begin{array}{l}1018.36 \\
\pm 600.69\end{array}$ \\
\hline $\operatorname{LL} 1\left(10^{5}\right)$ & $\begin{array}{c}3.46 \\
\pm 0.65\end{array}$ & $\begin{array}{c}3.57 \\
\pm 0.61\end{array}$ & $\begin{array}{l}12.47 \\
\pm 6.09\end{array}$ & $\begin{array}{l}1381.99 \\
\pm 207.50\end{array}$ \\
\hline $\operatorname{LL} 1\left(10^{6}\right)$ & $\begin{array}{c}6.04 \\
\pm 1.18^{*}\end{array}$ & $\begin{array}{c}2.57 \\
\pm 0.06\end{array}$ & $\begin{array}{l}14.32 \\
\pm 1.00\end{array}$ & $\begin{array}{l}2357.34 \\
\pm 13.19 *\end{array}$ \\
\hline Laminarina & $\begin{array}{c}2.37 \\
\pm 0.53\end{array}$ & $\begin{array}{c}2.43 \\
\pm 0.12\end{array}$ & $\begin{array}{l}13.20 \\
\pm 5.34\end{array}$ & $\begin{array}{l}2032.70 \\
\pm 151.80\end{array}$ \\
\hline
\end{tabular}

Bioensayo 2 = LL1 $\left(10^{4}, 10^{5}\right.$, o $\left.10^{6} \mathrm{UFC} / \mathrm{mL}\right)$ y Laminarina: $(0.05 \mathrm{mg} / \mathrm{L})$. $(*)$ denota diferencias significativas respecto al control $(p<0.05)$. 
$(p<0.05)$ comparados con el grupo control (Tabla 1). La cepa LL1 no generó diferencias significativas en la actividad SOD respecto al grupo control, presentando valores promedios desde $12 \pm 5$ a $18 \pm 3 \mathrm{U} / \mathrm{mg}$ (Tabla 2 ).

La actividad CAT proveniente de los hemocitos de $L$. vannamei del bioensayo 1 mostraron un patrón similar al observado en la actividad de SOD, registrando un incremento significativo en los camarones tratados con LAM (Tabla 1 ). La actividad de CAT obtenida de hemocitos de L. vannamei expuestos a la cepa LL1 revelan diferencia significativa $(p<0.05)$ al ser expuestos a la dosis de $1 \times 10^{6} \mathrm{UFC} / \mathrm{mL}$ con respecto al grupo control (Tabla 2).

\section{DISCUSIÓN}

La aplicación de levaduras en acuicultura se ha incrementado en los últimos años debido a su potencial benéfico (14). Tovar et al (15) reportaron que la incorporación del $1.1 \%$ de $D$. hansenii (cepa CBS 8339) en la dieta de larvas del pez Dicentrarcus labrax, incrementó en un $10 \%$ la supervivencia, duplicó el peso respecto al grupo control y redujo la proporción de malformaciones. Yang et al (14) observaron un incremento significativo en peso y supervivencia en juveniles de L. vannamei al ser alimentados con la levadura Rhodosporidium paludigenum incluida en la dieta al $1 \%$. Shupantharika et al (16) reportaron un aumento significativo en la actividad fenoloxidasa en hemocitos de $P$. monodon, tratado con $\beta$-glucano extraído de levadura de cerveza. En el presente estudio, el suministro de la levadura marina $D$. hansenii vía inmersión a una dosis de $1 \times 10^{6} \mathrm{UFC} / \mathrm{mL}$ generó un incremento de hemocitos circulantes, lo cual es considerado como un posible aumento en la capacidad de respuesta inmune del camarón en contra de virus y bacterias (17). Sajeevan et al (13) observaron que las células completas de levaduras proporcionaron a $F$. indicus un mayor CTH al usar productos purificados (glucanos) en su dieta. Esto sugiere que las células completas de levaduras pueden tener otros elementos que fortalezcan la respuesta inmune (aminoácidos, vitaminas, minerales, etc.). Otros trabajos reportan concentraciones similares que han demostrado el beneficio de los probióticos $(17,18)$. Sin embargo, se ha detectado que el CTH en crustáceos puede presentar variaciones importantes (19). Además, de que puede ser alterado por estrés, enfermedades y medio ambiente (20-22).

La mayoría de los probióticos propuestos en acuicultura pertenecen a los géneros
Aeromonas sp. Bacillus sp, Carnobacterium $\mathrm{sp}$, Flavobacterium $\mathrm{sp}$, Lactobacillus $\mathrm{sp}$, Pseudomonas sp, Vibrio sp, y a diferentes géneros de levaduras como Debaryomyces sp, Phaffia sp, Saccharomyces sp y Rhodosporidium sp (14). En el presente estudio, la utilización de $D$. hansenii a una concentración de $1 \times 10^{5}$ y $1 \times 10^{6}$ UFC/mL incrementaron el CTH, proteína y CAT. A pesar que no se incrementó significativamente la actividad SOD respecto al grupo control, en otros trabajos con probióticos se han reportado incrementos en la actividad de esta enzima asociando esta respuesta antioxidante como un incremento a la tolerancia a estrés oxidativo (17). La concentración de probióticos recomendada es entre $1 \times 10^{4}$ y $1 \times 10^{6} \mathrm{UFC} / \mathrm{mL}$ (18). En este estudio, la concentración de $1 \times 10^{4} \mathrm{UFC} / \mathrm{mL}$ de $D$. hansenii (cepa DH5) incrementó el contenido de proteína, lo cual podría indicar que otras inmunoproteínas podrían estar actuando en respuesta a la levadura (13). Downs et al (23) reportaron que el incremento en el contenido de proteína en hemocitos de camarón Palaemonetes pugio expuesto a estrés por temperatura se debió al incremento de diferentes proteínas relacionadas con el sistema inmunológico, principalmente citocinas y chaperoninas.

Un buen estado de salud de los organismos se ha sugerido que esta asociado a un valor alto de proteínas presente en los hemocitos y la hemolinfa, lo cual está relacionado con el estado inmune de los mismos $(21,24)$. Entre las proteínas detectadas en hemocitos y hemolinfa se han encontrado moléculas asociadas a la coagulación, metabolitos moduladores del sistema inmune, profenoloxidasa, proteínas de reconocimiento, peneidinas, peroxinecticas, hemocianina, entre otras $(1,25)$. Laria et al (21) observaron que el contenido de proteína en hemolinfa de $L$. schmitti no se ve afectada significativamente cuando los organismos se ven expuestos a diferentes dosis de formaldehido $(10,25$, y $50 \mathrm{mg} / \mathrm{L})$; mientras que Boonyaratpalin et al (19) observaron que los niveles de proteína en hemolinfa de $P$. monodon disminuyen por el efecto creciente del aflatoxina contenida en la dieta. Las dosis de $1 \times 10^{4} \mathrm{UFC} / \mathrm{mL}$ las cepas de levaduras marinas $D$. hansenii, DH5 y DH6, incrementaron significativamente los niveles de proteína en los hemocitos de L. vannamei.

El incremento en los niveles de actividad antioxidante en las células se relaciona con una rápida respuesta desintoxicante, reflejando en consecuencia la importancia de las enzimas SOD y CAT para remover el exceso de Sustancias Reactivas de Oxigeno (ROS) de la célula (25). 
Los valores de actividad SOD en hemocitos fueron incrementados significativamente en los camarones tratados con laminarina presentando un comportamiento similar en la actividad de CAT en los camarones tratados con laminarina y D. hansenii (cepa LL1). Las levaduras, además de aportar B-glucanos, mananooligosacaridos y otros componentes de la pared celular, son una fuente de antioxidantes, principalmente de $\operatorname{SOD}(7,8)$. Como era esperado, la laminarina generó un incremento de SOD y CAT en los hemocitos de los camarones tratados con este inmunoestimulante comercial (11), posiblemente la concentración de las cepas DH5 y DH6 de $D$. hansenii fue insuficiente para obtener un incremento en la actividad antioxidante. Liu et al (26) y Guertler et al (27) señalaron que la aplicación de laminarina en Astacus astacus, $F$. paulensis, L. schmitti y L. vannamei generó la activación de moléculas asociadas al sistema inmune de estos organismos y los niveles de SOD. Resulta interesante que la actividad SOD y CAT muestren comportamientos muy similares, mostrando la estrecha relación que existe entre estos dos componentes del sistema antioxidante.

Existe gran variedad de inmunoestimulantes comerciales en el mercado. Sin embargo, la extracción y purificación de inmunoestimulantes de los microorganismos es caro en términos de energía, tiempo y dinero. Es por ello que la utilización de levaduras en los cultivos, en lugar de inmunoestimulantes comerciales como los glucanos, puede reducir el costo de producción acuícola y conferir mayor aporte nutricional y protección contra enfermedades potenciales (13).

Los resultados obtenidos en la presente investigación mostraron que algunas cepas de $D$. hansenii pueden ser empleadas para incrementar el contenido de hemocitos circulantes en juveniles de camarón blanco e incrementar la actividad antioxidante. Sin embargo, para entender los procesos relacionados con el sistema antioxidante y el sistema de defensa del camarón, son necesarios mayores estudios involucrando la proteómica y técnicas moleculares para aportar resultados que auxilien en la búsqueda de medidas profilácticas que disminuyan la incidencia de enfermedades en los estanques de cultivo.

\section{Agradecimientos}

Este estudio fue financiado por el Consejo Nacional de Ciencia y Tecnología (CONACYT). SEP-CONACYT(25981). Agradecemos a Artemio Hernández Salmerón y a María Jesús Romero Geraldo por el apoyo brindado al presente trabajo.

\section{REFERENCIAS}

1. Aguirre-Guzmán G, Sánchez-Martínez JG, Campa-Córdova AI, Luna-González A, Ascencio F. Penaeid shrimp immune system: A Minireview. Thai J Vet Med 2009; 39: 205-215.

2. Zhen-Ming C, Liu G, Zhao S, Li J, Peng Y. Marine yeasts as biocontrol agents and producers of bio-products. Appl Microbiol Biotechnol 2010; 86:1227-1241.

3. Vici V, Bright Sing IS, Bhat SG. Application of bacterins and yeast Acremonium dyosporii to protect the larvae of Macrobrachium rosenbergii from vibriosis. Fish Shellfish Immunol 2000; 10:559-563.

4. Robles R, Sorgeloos P, Van Duffel $H$, Nelis $H$. Progress in biomedication using live foods. J Appl Ichthyol 1998; 14:207-212.

5. Miles DJC, Polchana J, Lilley JH, Kanchanakhan S, Thompson KD, Adams A. Immunostimulation of striped snakehead Channa striata against epizootic ulcerative syndrome. Aquaculture 2001; 195:1-15.
6. Gatesoupe FJ. Live yeasts in the gut: natural occurrence, dietary introduction, and their effects on fish health and development. Aquaculture 2007; 267:20-30.

7. Reyes-Becerril M, Salinas I, Cuesta A, Meseguer J, Tovar-Ramirez D, AscencioValle $F$, Esteban MA. Oral delivery of live yeast Debaryomyces hansenii modulates the main innate immune parameters and the expression of immune-relevant genes in the gilthead seabream (Sparus aurata L.). Fish Shellfish Immunol 2008a; 25:731-739.

8. Reyes-Becerril M, Tovar-Ramirez D, AscencioValle F, Civera-Cerecedo R, Gracia-López V, Barbosa-Solomieu V. Effects of dietary live yeast Debaryomyces hansenii on the immune and antioxidant system in juvenile leopard grouper Mycteroperca rosacea exposed to stress. Aquaculture 2008b; 280: 39-44.

9. Sukumaran V, Williams DL, Sajeevan TP, Rosamma P. Marine yeast glucans confer better protection than that of baker's yeast in Penaeus monodon against white spot syndrome virus infection. Aquac Res 2010; 41: 1799-1805. 
10. Muñoz M, Cedeno R, Rodriguez J, Knaap WPW, Mialhe E, Bachere E. Measurement of reactive oxygen intermediate production in haemocytes of penaeid shrimp, Penaeus vannamei. Aquaculture 2000; 191: 89-107.

11. Campa-Córdova AI, Hernández-Saavedra NY, Philippis R De, Ascencio F. Generation of superoxide anion and SOD activity in haemocytes and muscle of American white shrimp (Litopenaeus vannamei) as a response to $\beta$-glucan and sulphated polysaccharide. Fish Shellfish Immunol 2002; 12: 353-366.

12. Silakes S, Supamattaya K. Immunostimulant and vaccination in Black Tiger shrimp, Penaeus monodon Fabricius: II Production of vaccine from Vibrio harveyi and its application in Black Tiger shrimp, Penaeus monodon Fabricius. Songklanakarin J Sci Technol 2000; 22(Suppl.): 663-676.

13. Sajeevan TP, Lowman DW, Williams $D L$, Selven S, Anas A, Rosamma P. Marine yeast diet confers better protection than its cell wall component (1-3)-b-D-glucan as an immunostimulant in Fenneropenaeus indicus. Aquac Res 2009; 40: 1723-1730.

14. Yang SP, Wu ZH, Jian JC, Zhang XZ. Effect of marine red yeast $R$ hodosporium paludigenum on growth and antioxidant competence of Litopenaeus vannamei. Aquaculture 2010; 309: 62-65.

15. Tovar D, Zambonino-Infante J, Cahu C, Gatesoupe FJ, Vázquez-Juárez R. Influence of dietary live yeast on European sea bass (Dicentrarchus labrax) larval development. Aquaculture 2004; 234: 415-427.

16. Shupantharika $M$, Khunrae $P$, Thanardkit $P$, Verduyn C. Preparation of spent brewer's yeast $\beta$-glucans with potential application as an immunostimulant for black tiger shrimp, Penaeus monodon. Bioresource Technol 2003; 88: 55-60.

17. Macey BM, Coyne VE. Improved growth rate and disease resistance in farmed Haliotis midae through probiotic treatment. Aquaculture 2005; 245: 249-261.

18. Vine NG, Leukes WD, Horst K. Probiotics in marine larviculture. FEMS Microbiol Rev 2006; 30: 404-427.

19. Boonyaratpalin M, Supamattaya K, Verakunpiriya $V$, Suprasert D. Effects of aflatoxin B1 on growth performance, blood components, immune function and histopathological changes in black tiger shrimp (Penaeus monodon Fabricius). Aquac Res 2001; 32: 388-398.

20. Sritunyalucksana K, Gangnonngiw W, Archakunakorn S, Fegan D, Flegel TW. Bacterial clearance rate and a new differential hemocyte staining method to assess immunostimulant activity in shrimp. Dis Aquat Org 2005; 63: 89-94.

21. Laria RE, Cruz $Y$, Silveira R, Martínez M, González N. Effects of formalin on total haemocytes count and histopathological changes in the shrimp Litopenaeus schmitti (Pérez-Farfante \& Kensley 1997). Aquac Res 2008; 39: 1316-1321.

22. Sajeevan TP, Selven S, Rosamma P. b-Mercapto-ethanol-treated yeast showed better protection against white spot syndrome virus infection in Indian white shrimp Fenneropenaeus indicus. 2010. Aquac Res 2010; 41: e715-e718.

23. Downs C, Fauth JE, Woodley CM. Assessing the health of grass shrimp (Palaemonetes pugio) exposed to natural and anthropogenic stressors: A molecular biomarker system. Mar Biotechnol 2001; 3: 380-397.

24. Moreno I, Pichardo S, Jose A, GómezAmores L, Mate A, Vázquez CM, Camean AM. Antioxidant enzyme activity and lipid peroxidation in liver and kidney of rats exposed to microcystin-LR administered intraperitoneally. Toxicon 2005; 45:395-402.

25. Van de Braak K. Hemocytic defense in black tiger shrimp (Penaeus monodon). [Doctor degree thesis]. The Netherlands: Wageningen University. Wageningen Institute of Animal Science; 2002.

26. Liu $\mathrm{CH}$, Yeh $\mathrm{SP}$, Kuo $\mathrm{CH}$, Cheng $\mathrm{W}$, Chou $\mathrm{CH}$. The effect of sodium alginate on the immune response of tiger shrimp via dietary administration: Activity and gene transcription. Fish Shellfish Immunol 2006; 21:442-452.

27. Guertler C, Schleder DD, Barracco MA, Perazzolo LM. Comparative study of the intracellular superoxide anion production in different penaeid species through the NBT-reduction assay. Aquac Res 2010; 41: 1082-1088. 